Session 2513

\title{
Experiments in Waste Processing for Undergraduates
}

\author{
K. B. Lodge, R. A. Davis, D. Dorland and D. N. Baria \\ Chemical Engineering, University of Minnesota, Duluth, Minnesota 55812
}

\section{$\underline{\text { Summary }}$}

The emphasis on hazardous waste processing and pollution prevention in the Department of Chemical Engineering, University of Minnesota, Duluth (UMD), has been enhanced by a National Science Foundation Instrument and Laboratory Improvement (NSF ILI) grant. It funded the development of 6 new experiments for the laboratory classes and for demonstrations in lectures.

Two experiments provide the students with training in important techniques of analysis for waste streams. Wastewater is characterized by the measurement of the biochemical oxygen demand (BOD), a quantity vital for the assessment of discharges to receiving waters. We have built a simple flue with a burner in the laboratory to train the student in the use of a stack-gas analyzer. This measures the excess air, gas temperature and the concentrations of the common pollutants $\mathrm{CO}, \mathrm{CO}_{2}, \mathrm{NO}_{\mathrm{x}}$ and $\mathrm{SO}_{2}$ in flue gas.

Four experiments teach techniques of separation. A solvent, methanol, is removed from water in our custom-built continuous distillation unit. Salt is removed from brine with our purposebuilt reverse osmosis system. Ultrafiltration is used to separate dextran blue and riboflavin in an aqueous solution. Finally, heavy metal ions in water are replaced by benign cations in our ionexchange unit.

\section{$\underline{\text { Introduction }}$}

Located at the western end of Lake Superior, UMD is one of four coordinate campuses of the University of Minnesota system. It has an enrollment of approximately 8,000 students, undergraduate and graduate. Chemical Engineering was established as an undergraduate program in 1986. Our enrollment has increased steadily; in the fall of 1995 we had 162 majors and 30 students graduated in the spring of 1996 . Women and members of recognized minority groups make up about one quarter of our students. Graduates of our program compete successfully in the job market.

The program is accredited by ABET and is taught by 5 faculty. All students are required to complete a sequence of two courses in hazardous waste processing; topics include wastewater treatment, air pollution control, and solid waste management. This emphasis in the undergraduate program is complimented by the research interests of the faculty. These areas include beneficiation and utilization of low-rank coals, rheological properties of coal-water slurries, electrochemical engineering, measurement and estimation of thermodynamic properties of 
hydrophobic organic compounds (HOCs), distribution of HOCs in aquatic systems, chemical analysis for HOCs in environmental matrices, industrial wastewater treatment, ozonation, airpollution control, separations, and transport phenomena.

Our two-course laboratory sequence is directly enhanced by this work. Students start the sequence in the spring of their junior year and complete it in the fall of their senior year. There are two 3-hr lab sessions every week and students complete 4-6 experiments for which they prepare technical reports in various forms, complete written technical reports, memo reports and at least one oral report. We have about 2,000 sq ft in which to run this sequence, which also requires about $60 \%$ of our senior laboratory-services coordinator's time.

\section{The Experiments}

The basic approach is to design experiments that teach the principles of hazardous waste assessment and processing; these principles should also be fundamental to chemical engineering. For the assessment of pollution we have designed experiments for the measurement of biochemical oxygen demand (BOD) and for the analysis of flue gases. We introduce ideas for minimization of pollution and for recycling in the experiments on continuous distillation, ion exchange, reverse osmosis, and ultrafiltration.

\section{Biochemical Oxygen Demand (BOD)}

To run this experiment, we use an incubator (Fisher low temperature), a refrigerator, 2 dissolved oxygen measuring units (YSI 50B meter with YSI 5907 probe), 300-mL BOD bottles and commercially available seed (Fisher). The procedures we follow are described elsewhere ${ }^{1}$. We tested various samples and the seed-corrected 5-day BODs $\left(\mathrm{BOD}_{5}\right)$ are shown in Table I.

Table I. Five-day BODs of various samples

\begin{tabular}{ll}
\hline Sample & $\mathrm{BOD}_{5}, \mathrm{mg} / \mathrm{L}$ \\
\hline Potato cooking water -- from cook 1 & $>2500$ \\
Potato cooking water -- from cook 2 & 1000 \\
Dish water & $>850$ \\
Clothes wash water & 310 \\
Influent to wastewater treatment plant & 240 \\
Urban runoff & 20 \\
Lake Superior water & 1 \\
\hline
\end{tabular}

Ideas in kinetics may be reinforced by measuring the $\mathrm{BOD}_{2}$ and $\mathrm{BOD}_{5}$ at various temperatures. Measurements with various chemicals will demonstrate degradation properties; for example, methanol degrades faster than phenol. 


\section{Combustion}

We constructed a simple short flue (18 in. high, 3 in. ID) with a flue cap at the top; this we mounted in a walk-in fume hood. With a burner below the flue, we analyze the gases leaving the top with a portable stack gas analyzer (COSA 6000). The analyzer measures the levels of $\mathrm{O}_{2}$, $\mathrm{CO}_{2}, \mathrm{CO}, \mathrm{SO}_{2}$ and $\mathrm{NO}_{\mathrm{x}}$, the gas temperature, excess air, combustion efficiency; it will also do a semi-quantitative soot test. In figure 1 we show results obtained with this system.

\section{Ultrafiltration}

With a commercial low-volume hollow-fiber ultrafiltration (UF) apparatus (Amicon RA2000, cartridge S1Y10, pump LP-1), we can separate high-molecular-weight materials and small molecules in solution. For example, we separate riboflavin (vitamin $\mathrm{B}_{2}$, MW 376.4, Sigma Chemicals) from its aqueous solution with dextran blue (average MW $2 \times 10^{6}$, Sigma Chemicals). The compositions of the permeate and retentate we determine with a spectrophotometer (Spectronic Genesys 2). To do this we prepared separate solutions of riboflavin and dextran blue in $0.1 \mathrm{M} \mathrm{NaCl}$ and measured the absorbance of various dilutions (at the wavelengths of 445 $\mathrm{nm}$ for riboflavin and $619 \mathrm{~nm}$ for dextran blue).

The separation by ultrafiltration is characterized by the rejection coefficient, $\mathrm{R}$; this is defined in terms of concentration of the chemical in permeate, $C_{p}$, and the concentration of chemical in the retentate, $C_{r}: R=1-C_{p} / C_{r}$. Also, the recovery may be used and is defined in terms of mass of chemical in retentate, $M_{r}$, and the mass of chemical before filtration, $M_{i}$ : Recovery $=M_{r} / M_{i}$. We obtain complete separation of the riboflavin from the dextran blue; we show these results in figure 2. The spectral properties of these compounds are particularly convenient for this experiment, enabling the student to readily monitor the separation by eye (the permeate is yellow and the starting mixture is green). Further experiments include the determination of the relationship between the transmembrane pressure and the flux of the permeate.

\section{Ion Exchange}

In a self-contained ion exchange unit (Armfield), we can soften or demineralize hard water with a combination of columns, containing cationic and anionic exchange resins, in series. With the cationic resin in the hydrogen form, we monitor the breakthrough for the cationic resin by measuring the $\mathrm{pH}$ of the effluent. Hard water, prepared from $\mathrm{CaCl}_{2} \cdot 2 \mathrm{H}_{2} \mathrm{O}$, is a convenient feed; we measure the hardness of the effluent with a simple titration using a soap solution (APHA, LabChem, $1 \mathrm{~mL}$ soap solution $\equiv 1 \mathrm{mg} \mathrm{CaCO}_{3}$ ) whence the endpoint is marked by the appearance of scum on the liquid surface (an interesting application of the hard-water phenomenon!). We show the results of this experiment in figure 3.

\section{Reverse Osmosis}

We modified a commercial Reverse Osmosis unit (PUR Power Survivor 80II with PUR Model II-2 pump) for variable pressure operation. The unit is designed for low production (3 $\mathrm{gal} / \mathrm{hr}$ ), and is suitable for recreational purposes and emergency water purification. The feed and effluent stream compositions are determined from conductivity measurements using the salinity 
meter that comes with the unit. This experiment illustrates the reverse osmosis phenomenon by

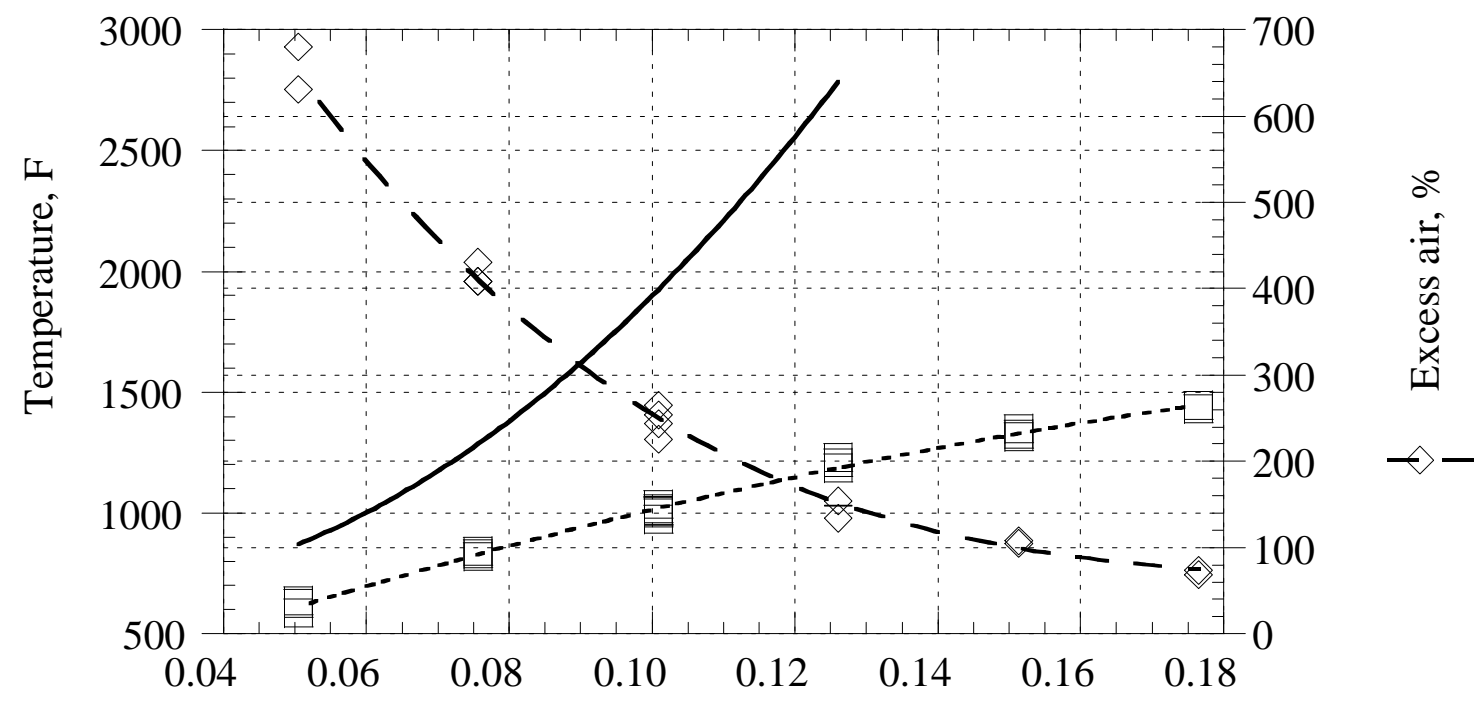

Natural gas flow, cu ft/min

-- - -- Flue gas temperature $\longrightarrow$ Adiabatic flame temperature

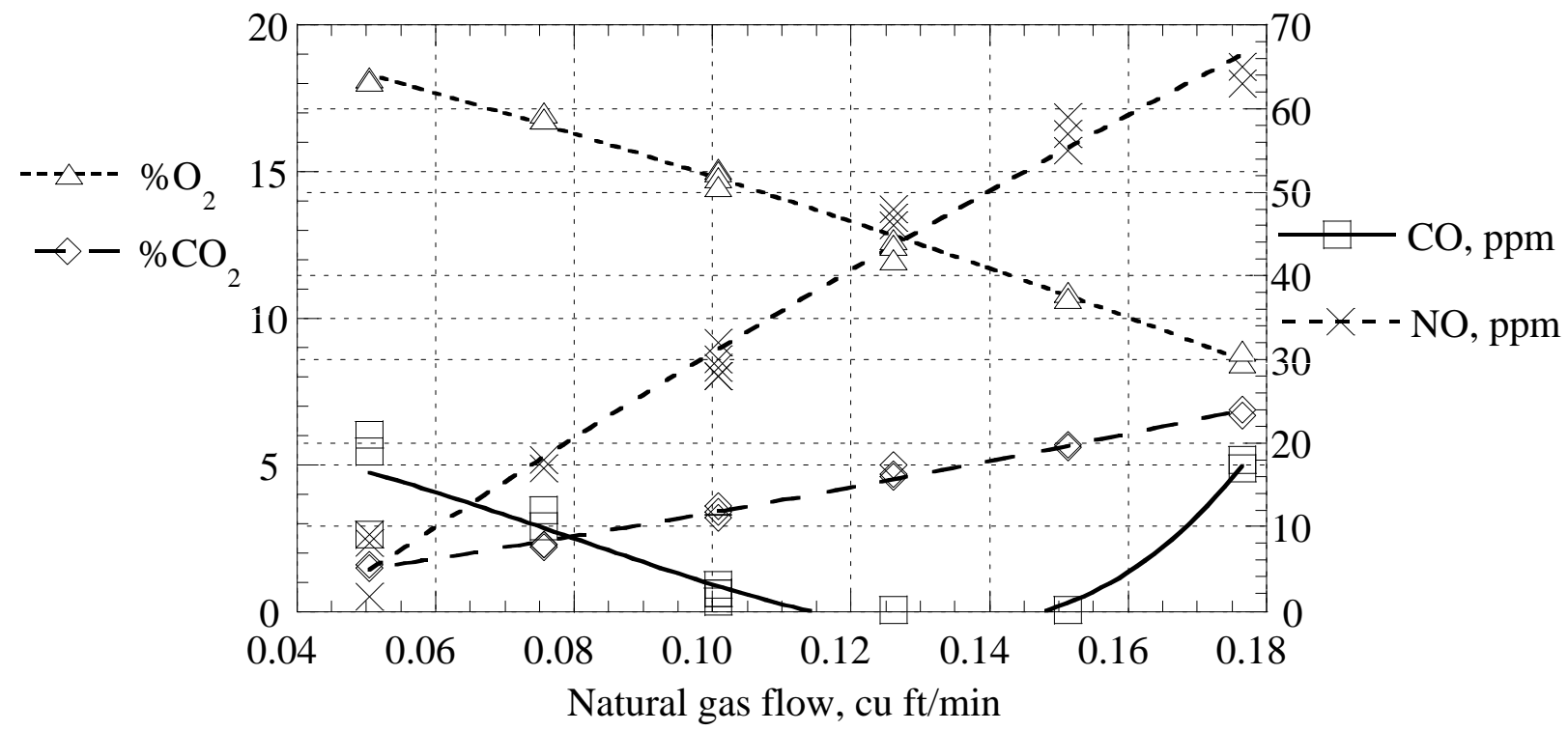

showing the effect of pressure and the rate of inlet water flow, containing $\mathrm{NaCl}$, on the retention rate of $\mathrm{NaCl}$.

Figure 1. Combustion

We used a Veriflow burner with an N-2 nozzle fed by natural gas (lab supply). No air was premixed with the gas so we had a diffusion flame ${ }^{2}$. We restricted the flow of air into the bottom of the flue with a tin plate containing a 1-in. square hole; the flame was positioned to go through the center of the hole with the top of the burner set about $1 \mathrm{in}$. below the tin plate. We passed the natural gas through a flow meter that we calibrated with a household gas meter. The tip of the 
probe of the gas analyzer we placed at the top of the flue just below the center of the cap. The results of the gas analyses are summarized above for various gas flows. In the top graph, we show the adiabatic flame temperature for the reaction:

$$
\mathrm{CH}_{4}+\text { Air } \rightarrow \mathrm{CO}_{2}+2 \mathrm{H}_{2} \mathrm{O}
$$

We detected no $\mathrm{SO}_{2}$ (detection limit $\leq 1 \mathrm{ppm}$ ) in this experiment.

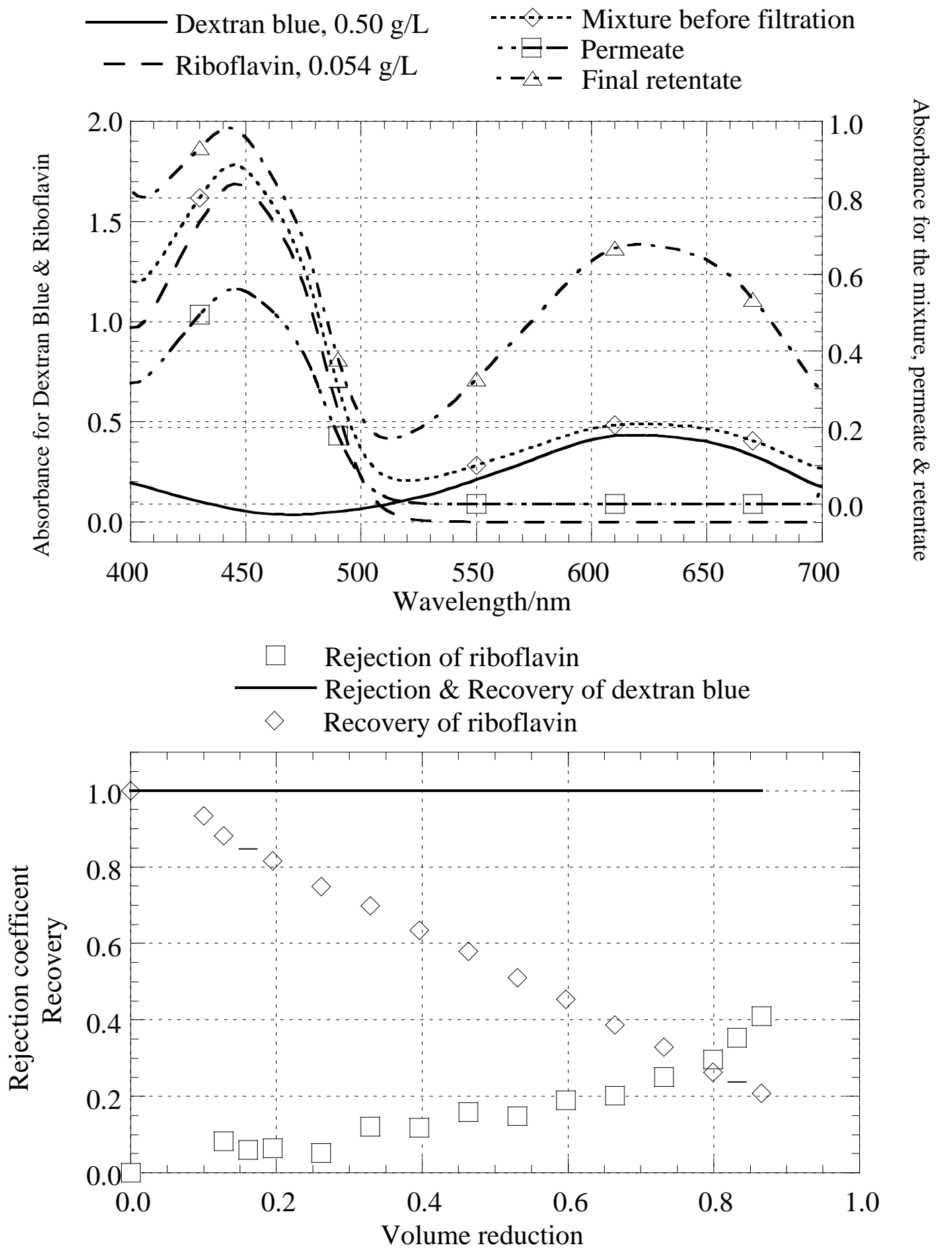


Figure 2. Ultrafiltration

In the supply reservoir of the ultrafiltration unit we mixed equal volumes of the two separate solutions of riboflavin and dextran blue in $0.1 \mathrm{M} \mathrm{NaCl}$. We set the flow rate of feed to the filter to give a transmembrane pressure of 15-16 psi, and we took samples of the permeate at various times and measured their compositions with the spectrophotometer. From these we calculate the rejection coefficient and the recovery.

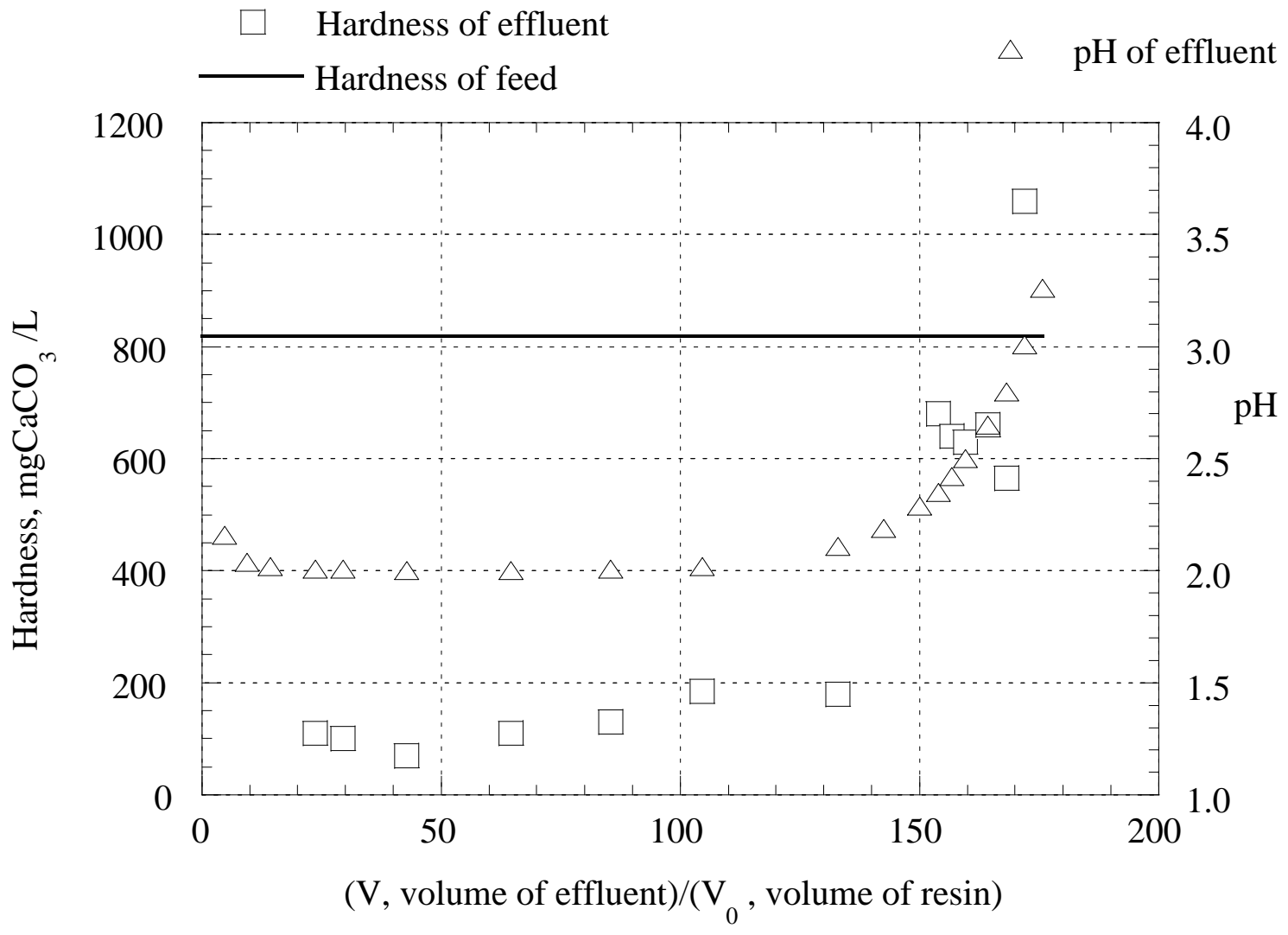

Figure 3. Ion Exchange

We pass artificially hardened water at $50 \mathrm{~mL} / \mathrm{min}$ through a cation exchange resin that has been prepared in the hydrogen form. The volume of wet resin is about $50 \mathrm{~mL}$ and the length of the column is $33 \mathrm{~cm}$. From $\mathrm{pH}$ and hardness measurements of the effluent, we determine the breakthrough point at about $\mathrm{V} / \mathrm{V}_{0}=140$. This corresponds to the resin having an exchange capacity of about 2 mequiv/mL of wet resin.

\section{Continuous Distillation}

We have constructed a bench-scale continuous distillation unit; the principal component is the glass-sieve plate column (ACE Glass) designed so the feed enters at the middle of the column. For feed mixtures of methanol and water of various compositions, we determine the column efficiency at steady state (constant reflux ratio, feed rate, overhead distillate rate and bottom product rate). To do this we measure the feed rate, overhead distillate rate and bottom product rate and the temperatures at the top and bottom of the column. The composition of the methanol- 
water mixtures we determine from both refractometry and pycnometry; this is necessary because the refractive index is not a single-valued function of composition within an important range of composition for this particular mixture.

\title{
Acknowledgments
}

We thank the National Science Foundation (Award \# 9451666) and the University of Minnesota, Duluth, for funding this work. We are indebted to D. Long for developing the BOD experiment and for his assistance throughout this work. For shop work, we thank Darrell Anderson. During the summer of 1996, three of our students, M. Sanders, S. Iqitdar and C. Merrifield, worked on the experiments described here.

\author{
$\underline{\text { References }}$ \\ 1. APHA, AWWA \& WPCF, Standard Methods for the Examination of Water and Wastewater, 18th edition, \\ American Public Health Association, Washington, DC, 1992. \\ 2. Barnard, J.A., Flame and Combustion, Englewood Cliffs, NJ, pp. 788-791 (1993).
}

\section{Biographical Information}

KEITH LODGE, assistant professor, has a Ph.D. in physical chemistry from the University of Sheffield, UK. His presently funded work includes physical, chemical and toxicological properties of chemicals of interest to the U.S. Air Force and the assessment of contaminated sediment. He has taught thermodynamics, hazardous waste processing, computational methods and unit operations laboratories.

RICHARD DAVIS, assistant professor, has a Ph.D. in chemical engineering from the University of California, Santa Barbara. His presently funded research includes the abatement of $\mathrm{NO}_{\mathrm{X}}$ during iron oxide pellet firing. He has taught fluid, heat, and mass transfer; reactor design; engineering economics; biochemical engineering and unit operations laboratories.

DIANNE DORLAND, professor and head of the Department of Chemical Engineering at UMD and registered professional engineer, has a Ph.D. in chemical engineering from West Virginia University. Recently, she had a large NSF grant with which she created a computer laboratory for engineering students. She has taught hazardous waste processing, stoichiometry and unit operations laboratories.

DORAB BARIA, professor, registered professional engineer and founding member of the Department of Chemical Engineering, has a Ph.D. in chemical engineering from Northwestern University. He has worked for the U.S. Atomic Energy Commission and was a faculty member at the University of North Dakota, Grand Forks. Dr. Baria has taught almost every course in the chemical engineering curriculum. 